\title{
Application of iTRAQ-Based Quantitative Proteomics Approach to Identify Deregulated Proteins Associated with Liver Toxicity Induced by Polygonum Multiflorum in Rats
}

\author{
Longfei Lin ${ }^{a}$ Hui Lia Hongmei Lin ${ }^{b} \quad$ Miao Zhang ${ }^{b}$ Changhai Qu ${ }^{b}$ Lei Yan \\ Xingbin Yin ${ }^{b}$ Jian $\mathrm{Ni}^{\text {b }}$
}

aInstitute Chinese materia medica china academy of Chinese medical sciences, Beijing, bSchool of Chinese material medica, Beijing University of Chinese Medicine, Beijing, 'Fengtai district community health center, Beijing, China

\section{Key Words}

Polygonum multiflorum • Proteomic • Hepatotoxicity • Biomarker • Mechanism • Oxidative phosphorylation

\begin{abstract}
:
Background/Aims: Clinical reports on adverse reactions that result from Polygonum multiflorum (PM) and its preparations, especially regarding liver injury, have recently received widespread attention. This study aimed to investigate the mechanism of hepatotoxicity induced by different PM extracts through iTRAQ quantitative proteomics. Methods: The different PM extracts were orally administrated for 90 days to rats, and the hepatotoxicity effect was evaluated through measurement of biochemical indexes, oxidative damage indexes and hematoxylin-eosin (HE) staining. Then, the hepatotoxicity mechanism was investigated by iTRAQ quantitative proteomics. Results: The results of biochemical and histopathological analyses showed that liver injury occurred in all groups of rats given by various PM extracts, which proved all of the PM extracts could induce hepatotoxicity. The hepatotoxicity mechanism may differ between the total extract group and the other groups through the results of biochemical indicators. The iTRAQ proteomics study showed that hepatotoxicity resulting from PM was mainly related to the abnormal activity of mitochondrion function-related oxidative phosphorylation pathways. Conclusion: This ITRAQ proteomics study revealed that the hepatotoxicity induced by PM is primarily related to the oxidative phosphorylation pathways. NADH dehydrogenase family proteins and Slc16a2 could be potential biomarkers of hepatotoxicity resulting from PM.

L. Lin and $\mathrm{H}$. li contributed equally to this work.




\section{Cellular Physiology Cell Physiol Biochem 2017;43:2102-2116 \\ \begin{tabular}{ll|l} 
and Biochemistry & $\begin{array}{l}\text { DOI: 10.1159/000484229 } \\
\text { Published onlIne: October 24, } 2017\end{array}$ & $\begin{array}{l}\text { C } 2017 \text { The Author(s). Published by S. Karger AG, Basel } \\
\text { www.karger.com/cpb }\end{array}$ \\
\hline
\end{tabular}}

\section{Introduction}

Polygonum multiflorum (PM) was first recorded in Kai Bao Ben Cao and is the roots of the polygonaceae plant Radix Polygoni Multiflori. The prepared polygonum multiflorum can enrich and benefit essence and blood, reinforce the kidneys and blacken beards and hair [1]. Raw PM can relieve toxicity, stop malaria and moisten the intestines. Clinical reports on adverse reactions due to PM, particularly liver injury, and its preparations have recently received significant attention. The China Food and Drug Administration (CFDA) published the following series of reports on this problem: "Notice issued by the CFDA General Office on the Modification of Six TCM Oral Preparations Containing PM Yangxu Shengfa Capsules"; "Notice Issued by the CFDA General Office on Stipulations with respect to Strengthening the Supervision over Health Foods Containing PM" and "Adverse Drug Reactions Information Bulletin (61 $\left.{ }^{\text {st }}\right)$ Focusing on Liver Damage Risk from Orally ingesting PM and its Drug Preparations". Such reports indicate that the following situations can increase the risk of liver damage: consuming large doses and long-term continuous medications; consuming raw PM; patients with liver damage; and taking in conjunction with other liver-damaging drugs. The Medicines and Healthcare Products Regulatory Agency (MHRA) also released relevant information regarding adverse reactions to PM. Liver injury, especially acute liver injury, due to PM consumption has recently been reported worldwide [2-4].

Although PM toxicity is of great concern, the hepatotoxic components and mechanisms remain unknown. In this study, the ingredients in PM were analysed by type via separating PM into various extracts, including the dichloromethane (DCM) extract (the content mainly including free anthraquinone), water extract (the content mainly including tannins and polysaccharide), 30 ethanol extract (mainly composed of polyhydroxystilbene, such 2, 3,5, 4 '-tetrahydroxystilbene-2-0- $\beta$-glucoside, TSG) and 70 ethanol extract (mainly composed of combined anthraquinone, such as emodin-8-0- $\beta$-D-glucopyranoside, EDG). In addition, the hepatotoxicity of these extracts was investigated in vivo, and the hepatotoxicity mechanism was investigated by iTRAQ quantitative proteomics. The results of this study provide insight into the rational clinical use of PM and its patented drug forms.

\section{Materials and Methods}

\section{Chemicals}

Methanol (HPLC-grade) was purchased from Fisher Scientific (Waltham, MA, USA). HPLC-quality water was obtained from a Cascada ${ }^{\mathrm{TM}}$ IX-water Purification System (Pall Co., Port Washington, NY, USA). Ammonia was purchased from Guangdong Xilong Chemical Co., Ltd. (Shenzhen, China). PM was provided by Beijing Tongrentang Co., Ltd. (Beijing, China). Standard samples of emodin and TSG were purchased from Shanghai Standard Biotech Co., Ltd. (Shanghai, China). EDG was purchased from Shanghai Yuanye Bio-Technology Co., Ltd. (Shanghai, China). Carbamide and CHAPS were purchased from Bio-Rad Laboratories, Inc. (California, USA). A Protein Assay Kit was purchased from Thermo Scientific (Thermo Fisher Scientific Corp., MA, USA). Thiourea and bovine serum albumin were purchased from Sigma-Aldrich Corporation (St. Louis, USA). Reducing reagent, cysteine-blocking reagent and dissolution buffer in the iTRAQ kit were purchased from AB Sciex Corporation (Washington, D.C., USA).

\section{Preparation of PM extracts}

PM was decocted in 10 -fold of $70 \%$ ethanol three times, and then with 10 -fold of water once for 1.5 $\mathrm{h}$ each. The resulting filtrates were combined and concentrated to dryness under reduced pressure, which yielded the total extract. Another PM was decocted by the above methods, combined with the $70 \%$ ethanol extracts, and extracted with the same volume of DCM 5 times after being recovered and concentrated. Then, the DCM solution was combined and concentrated to dryness under reduced pressure, which yielded the DCM extract (primary content of free anthraquinone). The portion extracted by DCM was separated by HPD-300 macroporous resin, combined the water and 10\% ethanol elution to yielded the water extract 


\section{Cellular Physiology Cell Physiol Biochem 2017;43:2102-2116 \\ \begin{tabular}{ll|l} 
DOI: 10.1159/000484229 & $\begin{array}{l}\text { @ } 2017 \text { The Author(s). Published by S. Karger AG, Basel } \\
\text { www.karger.com/cpb }\end{array}$ \\
\hline
\end{tabular}}

Lin et al.: iTRAQ-Proteomics of Liver Toxicity Induced by Polygonum Multiflorum in Rats

(primary content of tannins and polysaccharide); the 30\% ethanol elution yielded the 30 ethanol extract (primarily composed of polyhydroxystilbenes, such TSG); and the 70\% ethanol and 95\% ethanol elutions were combined to yield the 70 ethanol extract (primarily composed of combined anthraquinones, such as EDG).

\section{Analysis of the main ingredients in different extracts of PM}

The contents of emodin, TSG and EDG in the different extracts of PM were analysed using a Prominence LC-20A HPLC system equipped with a SPD-20A UV-Detector (SHIMADZU Corporation, Japan). The different extracts of PM and the three standard compounds were accurately weighed and treated with ultrasound for $30 \mathrm{~min}$ after adding the specified volume of methanol. The supernatant was obtained and filtered using a $0.22 \mu \mathrm{m}$-micropore filter, and then the compositions in the extracts were measured by HPLC.

The analysis of the main ingredients in different extracts of PM (the concentration of the crude herbs was equivalent in each sample) was performed using a Waters Acquity Ultra Performance LC system coupled with a Xevo G2 Q-TOF mass spectrometer equipped with an electrospray ionization source (Waters Corp., Milford, MA, USA). Negative ion ionization was used to detect the compounds and had a higher sensitivity than positive mode. The cone gas and the source temperature were set to $50 \mathrm{~L} / \mathrm{h}$ and $120^{\circ} \mathrm{C}$. The capillary voltage was $2.5 \mathrm{kV}$. High-purity nitrogen served as both the nebulizing and dry gas. The desolvation temperature was held at $350^{\circ} \mathrm{C}$, and the gas flow was $600 \mathrm{~L} / \mathrm{h}$. The full-scan range was from 50 to $1200 \mathrm{~m} / \mathrm{z}$.

\section{Hepatotoxicity of different extracts of PM}

Principles of laboratory animal care were followed, and all procedures were conducted according to the guidelines established by the National Institutes of Health. Every effort was made to minimize animal suffering. This study was approved by the Animal Ethics Committee at the Beijing University of Chinese Medicine. A total of 120 male Sprague-Dawley rats (160 $10 \mathrm{~g}$ ) were used as supplied by Beijing Vital River Laboratory Animal Technology (Beijing, China). All rats were maintained in plastic cages under standard environmental conditions at $26 \pm 2^{\circ} \mathrm{C}$ with a relative humidity of $50 \pm 10 \%$ and a dark/light cycle of $12 \mathrm{~h}$. The rats were fed a standard rat diet and water ad libitum. The rats were used for experimentation after 7 days of acclimatization. All experiments were performed during the day. All rats were randomly divided into six groups (20 animals/per group). The rats of the sixth group (control group) were fed with an equal volume of blank solvent only. The rats of groups 1-5 were treated with different extracts of PM (total extract, water extract, 30 ethanol extract, 70 ethanol extract and DCM extract in sequence) at doses of $6 \mathrm{~g} / \mathrm{kg}$ body weight per day (determined by the quantity of crude material). The dried extract was re-dissolved and dispersed in 0.5\%CMC-Na solution and then intragastrically administered to rats for 90 days; rats were weighed and their diet was recorded every day. Fasting for $12 \mathrm{~h}$ was required before the final administration, then anaesthesia was induced by intraperitoneal injection of chlorine hydrate. Blood was sampled from the aorta abdominalis, and the serum and plasma were separated and stored at $-80^{\circ} \mathrm{C}$ for measurement of biochemical indexes (AST, ALT, TBIL, DBIL, IBIL, TP, ALB, GLB, ALP, GLU, TG, CHO, HDL, LDL and LDH). The liver tissues were rapidly frozen in liquid nitrogen and stored at $-80^{\circ} \mathrm{C}$ for evaluating the oxidative damage indexes (SOD and GSH-Px) and performing the proteomics study. Another sample of liver tissue was collected for pathology examination after fixing in formalin, trimming, embedding in paraffin, conventionally incising and staining with HE. All biochemical indexes and oxidative damage indexes were expressed as the mean \pm SD. The data were subjected to ANOVA to evaluate the significance of the changes using SPSS 17.0 software, $P$ value less than 0.05 is considered as statistically significant.

\section{The iTRAQ quantitative proteomics study}

Protein extraction, digestion and iTRAQ labelling. For each group, each individual sample of equal tissue weights was mixed, and then the proteins were extracted from the mixed samples. One millilitre of lysis buffer containing $7 \mathrm{M}$ urea, $2 \mathrm{M}$ thiourea and 0.1\% CHAPS was added to the mixed samples, followed by tissue homogenization and sonication. After centrifugation at $15,000 \mathrm{~g}$ for $20 \mathrm{~min}$ at $4{ }^{\circ} \mathrm{C}$, the supernatant was collected and transferred to a fresh tube. Protein concentrations were established by the Bradford assay following the manufacturer's protocol. In addition, then the protein samples $(200 \mu \mathrm{g})$ were digested with trypsin solution $\left(4 \mu \mathrm{g}\right.$ ) overnight at $37{ }^{\circ} \mathrm{C}$ and labelled with iTRAQ reagents (AB SCIEX, Massachusetts, USA) as follows: total group (113 tag), water group (114 tag), 30 ethanol group (117 tag), 70 ethanol group (118 tag), DCM group (119 tag) and control group (121 tag) post-injury according to the manufacturer's instructions. Then, the six labelled samples were pooled, centrifuged and dried. 


\section{Cellular Physiology Cell Physiol Biochem 2017;43:2102-2116 \\ \begin{tabular}{ll|l} 
and Biochemistry & $\begin{array}{l}\text { DOI: 10.1159/000484229 } \\
\text { Published onlıne: October 24, } 2017\end{array}$ & $\begin{array}{l}\text { C } 2017 \text { The Author(s). Published by S. Karger AG, Basel } \\
\text { www.karger.com/cpb }\end{array}$ \\
\hline
\end{tabular}}

LC and MS/MS analysis. The peptide mixture was re-dissolved in solution A (Solution A: 98\% $\mathrm{ddH}_{2} \mathrm{O}, 2 \%$ acetonitrile, $\mathrm{pH} 10.0$ ) and then fractionated by high pH separation using a RIGOL L-3000RPHPLC system, and $100 \mu \mathrm{g}$ of the mixture was then desalted and fractionated using a Durashell- $\mathrm{C}_{18}$ reverse phase column ( $4.6 \mathrm{~mm} \times 250 \mathrm{~mm}, 5 \mu \mathrm{m}$, Agela). Next, $98 \%$ acetonitrile with $2 \% \mathrm{ddH}_{2} \mathrm{O}$ in solution B was added, and the $\mathrm{pH}$ was adjusted to 10.0 with ammonium hydroxide. The column flow rate was maintained at $700 \mu \mathrm{L} / \mathrm{min}$, and the column temperature was maintained at room temperature. After separation, the fractions were re-suspended in $20 \mu \mathrm{L}$ solution C (solution C:0.1\% formic acid and $2 \%$ methanol in water), separated by nano-LC and analysed by on-line electrospray tandem mass spectrometry.

Nano-LC-MS/MS experiments were performed using an EASY-nLC 1000 coupled with Q-Exactive System (Thermo Fisher Scientific Corp., MA, USA). Peptides were loaded on a $75 \mu \mathrm{m} \times 120 \mathrm{~mm}$ nanocolumn (EASY-Spray column, $\mathrm{C}_{18}, 3 \mu \mathrm{m}$ ), and $0.1 \%$ formic acid acetonitrile solution was used as solvent D. The flow rate was set at $350 \mathrm{~nL} / \mathrm{min}$, and the injection volume was $10 \mu \mathrm{L}$. The spray voltage was $2.1 \mathrm{kV}$, the capillary temperature was set to $250^{\circ} \mathrm{C}$, the ion source was the EASY-Spray source, and the DP was 100 . The Q-Exactive mass spectrometer was operated in data dependent mode to switch automatically between MS and MS/MS acquisitions. Surveys of the full-scan MS spectra (m/z 350-1800) were acquired with a mass resolution of $70000 \mathrm{FWHM}$, followed by 15 sequential high-energy collisional dissociation MS/MS scans with a resolution of 17500 FWHM.

Proteomic data analysis and bioinformatics. All raw files generated by the Q-Exactive system were converted to $\mathrm{m} / \mathrm{z}$ XML and MGF files using the MS convert module in the Trans-proteomic Pipeline. All MGF files were searched using Proteome Discoverer1.3 (Thermo Fisher Scientific Corp., MA, USA) against a human database provided by The Universal Protein Resource (http://www.uniprot.org/uniprot). For identifying proteins whose expression was significantly altered in diseased liver tissues (compared with its non-diseased liver tissues), proteins were accepted using a false discovery rate (FDR) $<1 \%$ and protein confidence $>99 \%$. We set a threshold of iTRAQ ratios to define differentially expressed proteins. The proteins were considered to be differentially expressed if the iTRAQ ratio was $>1.2$ or $<0.833$. This cut-off value is recommended in the literature $[5,6]$ and was used to obtain more comprehensive information regarding the differences in the proteomic analysis.

Biological process, molecular function, cellular component and pathway enrichment analyses of the identified proteins were evaluated using the bioinformatics analysis tool DAVID (http://david.abcc.ncifcrf. gov). Functional interaction networks were identified using the STRING software (http://stringdb.org/). Polypeptides that showed a change in expression levels were located in the pathway biological maps using KEGG (http://www.genome.jp/kegg/).

\section{Western blot analysis}

Western blot is a commonly used method in molecular biology, biological chemistry and immunological genetics that can be used in qualitative and semi-quantitative analyses of protein. Colouring location and depth can be analysed to evaluate the expression of protein in cells or tissues. In this study, proteins extracted from liver tissues of each group were used in western blotting to validate the iTRAQ results. The control group and the administrated group samples were used in western blotting analyses, each sample was repeated for three times. $30 \mu \mathrm{g}$ of proteins from each sample were first resolved in the $12 \%$ SDS-PAGE gel and then transferred onto a nitrocellulose membrane (Bio-Rad, Hercules, CA, USA). The membrane was rinsed with PBS and the non-specific binding sites were blocked in a solution of 5\% non-fat milk in PBST $\left(0.05 \%\right.$ Tween 20 in PBS) for $2 \mathrm{~h}$ at $37^{\circ} \mathrm{C}$, followed by three times washes in PBST. The membrane was first incubated with rabbit $\alpha_{2}$-M antibody (1:5, 000 dilution) overnight and then washed in PBST buffer. An enhanced ECL detection system was used for visualization of NDUFV3 and SLC16A2 (Lyophilized powder, OriGene Technologies, Beijing, China).

\section{Results}

The compositions of the various PM extracts

PM was separated by dual ethanol-water extraction to ensure that all of the components of PM were extracted to the highest degree. The emodin, TSG and EDG contents in five extracts of PM are summarized in Table 1, which were calculated via standard external methods. The 
Table. 1. The contents (mg/g) of three major compounds in the five extracts

\begin{tabular}{lcccc}
\hline Group & Dried extract rate (\%) & Emodin $(\mathrm{mg} / \mathrm{g})$ & TSG(mg/g) & EDG(mg/g) \\
\hline Total & 36.67 & 2.23 & 96.46 & 10.02 \\
Water & 23.33 & 0.05 & 5.35 & 0.72 \\
30 Ethanol & 7.33 & 0.24 & 355.51 & 8.94 \\
70 Ethanol & 2.35 & 1.65 & 242.74 & 126.77 \\
DCM & 0.54 & 113.22 & 5.22 & 2.41 \\
\hline
\end{tabular}

Table 2. The peak area of major compounds in different parts of Polygonum multiflorum

\begin{tabular}{|c|c|c|c|c|c|c|c|}
\hline Compounds & $t_{R}$ & {$[\mathrm{M}-\mathrm{H}]^{-}$} & Total & Water & 30 & 70 & DCM \\
\hline Glucosyl-glucose & 0.73 & 341.108 & 517618 & 599876 & 10554 & 14518 & 5893 \\
\hline Citric acid & 0.82 & 191.019 & 485946 & 426209 & 850 & 1316 & 119 \\
\hline Gallic acid & 1.43 & 169.0134 & 112862 & 74747 & 77235 & 4504 & 7 \\
\hline Catechin & 3.61 & 289.0709 & 40251 & 6539 & 106684 & 106 & -- \\
\hline Tetrahydroxystilbene-0-di-hex & 4.14 & 567.1722 & 4402 & -- & -- & -- & -- \\
\hline L-epicatechin & 4.67 & 289.071 & 7922 & 1082 & 59340 & -- & -- \\
\hline Tetrahydroxystilbene-0-hex & 5.78 & 405.1177 & 22323 & 1570 & 27044 & 51 & -- \\
\hline Catechin-O-furanoside & 6.66 & 421.1136 & 9840 & -- & 3089 & -- & -- \\
\hline Tetrahydroxystilbene-0-di-hex & 7.01 & 567.1724 & 20437 & -- & 8061 & -- & -- \\
\hline Tetrahydroxystilbene-0-di-hex & 7.54 & 567.1719 & 12319 & -- & -- & -- & -- \\
\hline TSG & 8.26 & 405.1184 & 2362742 & 297830 & 2210873 & 975953 & 15841 \\
\hline Tetrahydroxystilbene-0-(galloyl)-hex & 9.26 & 557.1292 & 68920 & -- & 6237 & -- & -- \\
\hline Hydroxymusizin-0-hex-pen & 9.79 & 525.1601 & 5816 & -- & 841 & -- & -- \\
\hline Tetrahydroxystilbene-0-(galloyl)-hex & 10.45 & 557.1294 & 28388 & 287 & 48377 & 1369 & -- \\
\hline Emodin-O-glc & 11.51 & 431.0976 & 77012 & 3045 & 29894 & 54232 & -- \\
\hline Tetrahydroxystilbene-O-(galloyl)-hex & 11.92 & 557.1297 & 17246 & -- & 4522 & -- & -- \\
\hline Emodin-O-hex-sulphate & 12.51 & 511.0544 & 115147 & 1383 & 107008 & 47098 & -- \\
\hline $\mathrm{N}$-trans-feruloyl tyramine & 12.67 & 312.123 & 8924 & -- & -- & 410 & 13265 \\
\hline N-trans-Feruloyl-3-methyldopamine & 13.01 & 342.1332 & 3095 & -- & -- & -- & 6306 \\
\hline Torachrysone-8-0-glc & 13.57 & 407.1342 & 378708 & 14185 & 25097 & 193616 & 20799 \\
\hline EDG & 14.01 & 431.0975 & 867266 & 121229 & 348206 & 833368 & 10186 \\
\hline Anthraquinone-0-glc & 14.85 & 445.1132 & 11615 & -- & -- & 8438 & -- \\
\hline Emodin-0-(acetyl)-hex & 14.87 & 473.1102 & 21828 & 61 & 258 & 280 & -- \\
\hline Emodin-0-(malonyl)-hex & 14.87 & 517.0981 & 95921 & 2186 & 3132 & -- & -- \\
\hline Anthraquinone & 15.13 & 283.0602 & 148992 & 16702 & 7768 & 112781 & 7309 \\
\hline Anthraquinone-0-glc & 15.86 & 445.1135 & 5466 & 225 & -- & -- & -- \\
\hline Anthraquinone & 16.12 & 283.061 & 5001 & -- & -- & -- & -- \\
\hline Anthraquinone & 17.23 & 283.0605 & 14595 & 105 & 2 & 32 & 8291 \\
\hline Anthraquinone & 17.56 & 269.0448 & 20371 & 54 & 1222 & 15386 & 11 \\
\hline Anthraquinone & 17.63 & 283.0603 & 34880 & 793 & 349 & 1443 & 23623 \\
\hline Emodin & 18.74 & 269.0447 & 934867 & 66334 & 61318 & 131692 & 805307 \\
\hline Anthraquinone & 19.32 & 283.0607 & 31945 & 1245 & -- & -- & -- \\
\hline
\end{tabular}

results indicate that the content of each component was relatively low in the water extract; TSG was mainly present in the 30 ethanol extract; emodin was mainly present in the DCM extract, and EDG was mainly present in the 70 ethanol extract.

The analysis results of the main ingredients [7] and the peak areas for different extracts of PM by UHPLC-Q-TOF/MS are shown in Table 2. The base peak intensity chromatogram is shown in Fig. 1. The content of the DCM extract was primarily free anthraquinones, such as emodin and other free anthraquinones. The 70 ethanol extract was mainly composed of combined anthraquinone, such as EDG, anthraquinone-0-glc, and torachrysone-8O-glc. The 30 ethanol extract was mainly composed of polyhydroxystilbenes, such TSG, tetrahydroxystilbene-0-(galloyl)-hex, tetrahydroxystilbene-0-di-hex and others. The content of the water extract was mainly composed of tannins and polysaccharide, such as 


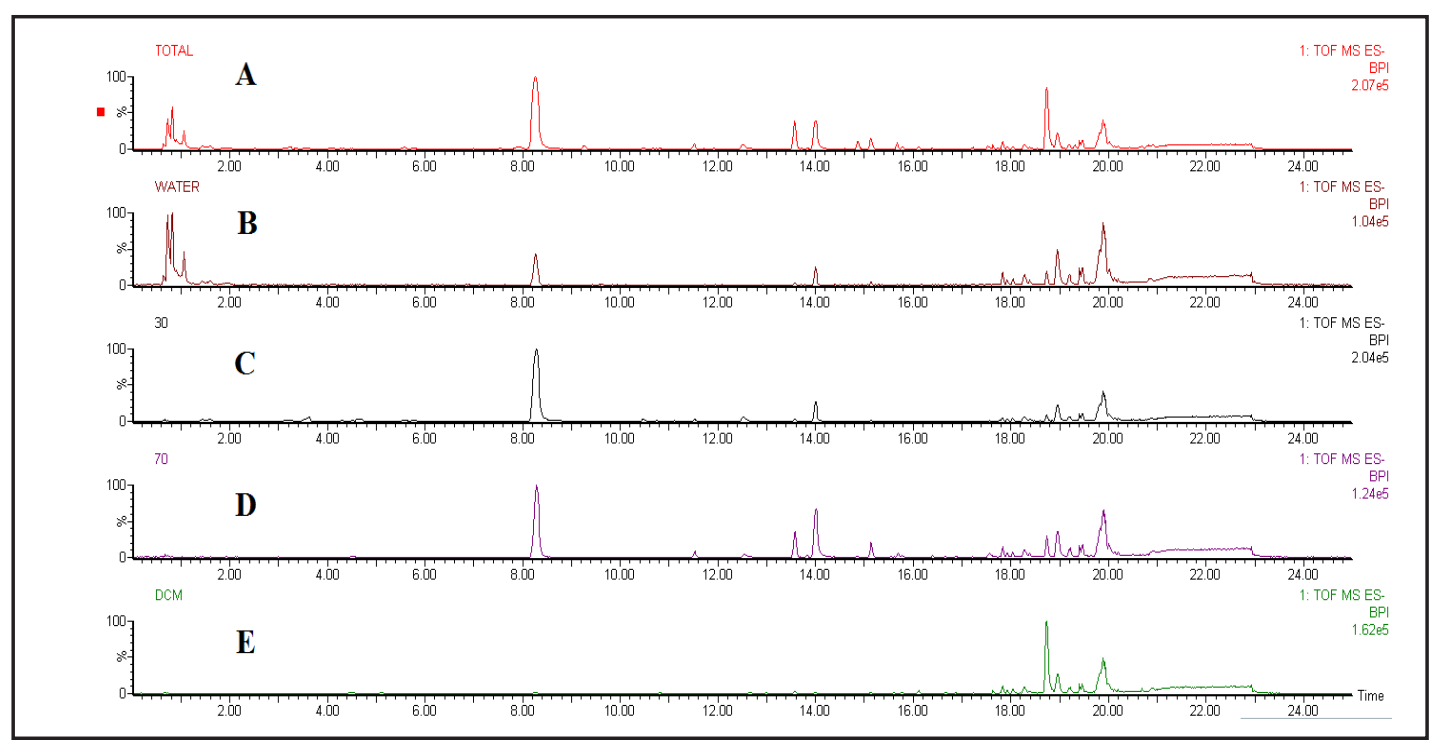

Fig. 1. Base peak intensity chromatogram in the negative mode of Total (A), Water (B), 30 Ethanol (C) , 70 Ethanol (D) and DCM (E) as determined by U-HPLC-Q-TOF/MS.

glucosyl-glucose, citric acid, gallic acid and other tannins or polysaccharides that could not be detected by UHPLC-Q-TOF/MS. Complex composition is one of the biggest characteristics of traditional Chinese medicine, and such extraction and separation methods can effectively classify the constituents in PM.

\section{Hepatotoxicity of different extracts of PM}

The changes in serum biochemical and antioxidant indexes. Table 3 summarizes the results of the biochemical analyses of rats treated with different extracts of PM. The results show that serum AST, ALT, ALP and LDH levels of the water, 30 ethanol, 70 ethanol and DCM extract-treated groups were significantly higher than those of the control group. The serum AST and ALT levels of the 30 ethanol extract group exhibited the greatest increases, corresponding to up to 5 times the levels of the control group. AST and ALT secretion specifically reflect the function of liver cells. Serum ALT activity is the most frequently relied upon laboratory indicator of hepatotoxicity. As a general rule, ALT levels 3 times greater than normal indicate liver injury. The above results indicate that liver injury occurred in the four groups of rats. However, another phenomenon observed was that the serum AST, ALT, ALP and LDH levels of the total extract group were not significantly higher than those of the control group, and the serum TBIL and DBIL levels increased more significantly. This indicated that the mechanism of liver injury in the total extract group differed from the other groups, such as via bile duct blockage, jaundice hepatitis, and haemolytic diseases, although the specific mechanism requires further study.

The blood glucose values of rats of the total extract group and water extract group were significantly higher than the other groups but their dietary intake was equivalent. This result indicated that these two groups of rats experienced abnormal glucose metabolism-related liver function (such as glycolysis and tricarboxylic acid cycle). The levels of TG, CHO and HDL in the serum of the total extract group rats were lower than those of the control group, which indicated possible abnormal lipid metabolism in the liver. The LDL levels and CHO levels in the serum of the water, 30 ethanol and 70 ethanol extract groups were statistically significantly higher than those of the control group, thus indicating abnormal synthesis and transport processes of $\mathrm{CHO}$ in the livers of these three groups.

Superoxide dismutase (SOD) is a free radical scavenger that can promote the transformation of free radicals into hydrogen peroxide and oxygen to eliminate the free radicals produced in the process of inflammation. Thus, SOD has a strong anti-inflammatory 
effect. Liver SOD and GSH-P were significantly reduced in all treated groups, indicating that the mechanisms of liver injury in these groups may be related to peroxide damage.

Histopathological analysis. As shown in Fig. 2, liver tissue sections of the control group of rats revealed clearly and completely outlined liver lobules, neatly arranged liver cells, no obvious degeneration or necrosis, a clearly visible hepatic sinus and no obvious congestion expansion, which were observed under a microscope after HE staining. Cell swelling, ballooning of degenerating cells, vacuolar degeneration accompanied by spotty necrosis of cells, focal infiltration of inflammatory cells in the central vein, and adipose tissue degeneration were observed in pathological liver tissue sections of the other groups of rats. This result indicates that the parenchymal liver injury has been produced.

The results of biochemical, antioxidant indexes and histopathological analysis showed that liver injury occurred in all 5 groups of rats after administration of the various extracts of $\mathrm{PM}$ and also indicated that all of the extracts (total, water, 30 ethanol, 70 ethanol and DCM extracts) could induce hepatotoxicity. The levels of ALT, AST and ALP indicate that the hepatotoxicity of the 30 ethanol group was the highest. Furthermore, the levels of TBIL and DBIL infer that the hepatotoxicity mechanism may differ between the total extract group and the other groups.

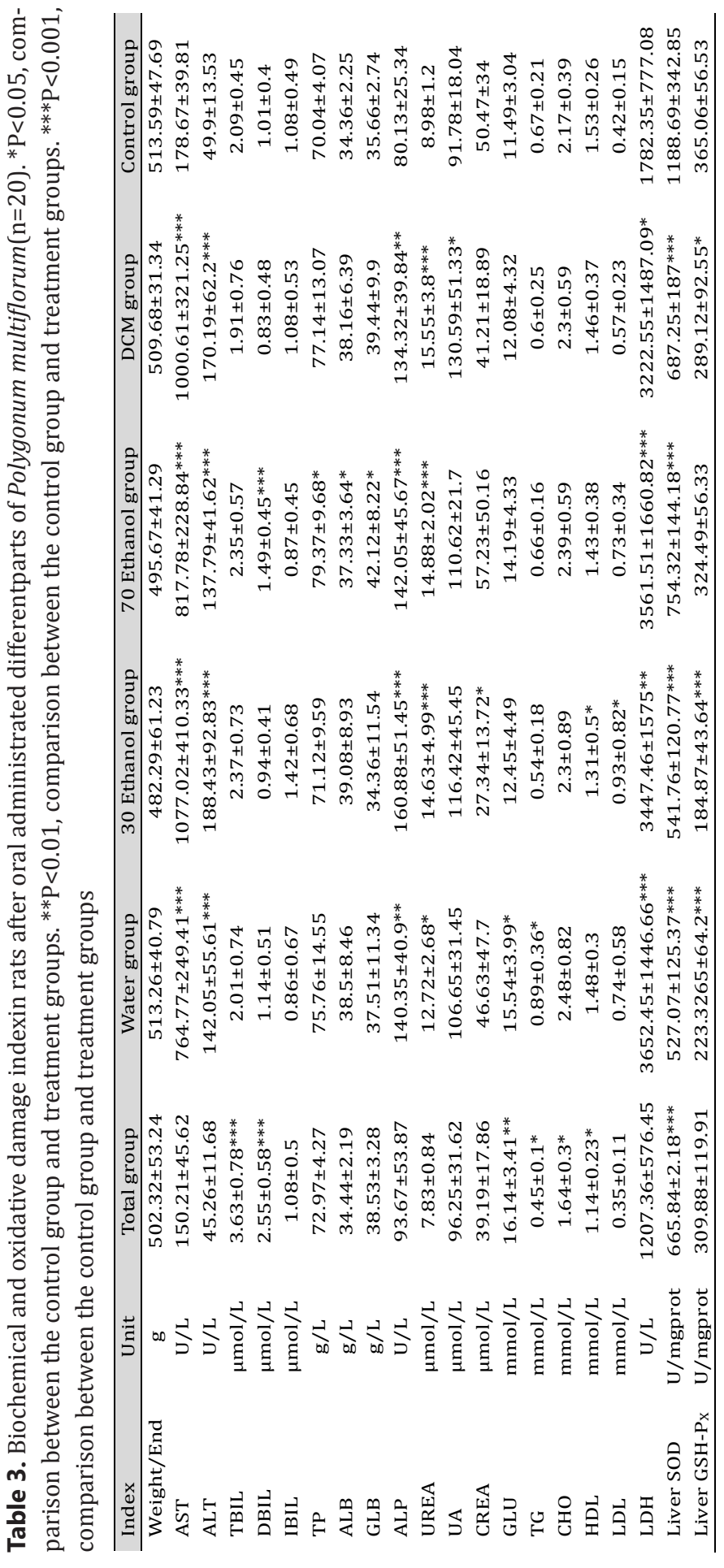

\section{Results of ITRAQ proteomics}

Gene ontology (GO) analysis. All MS/MS spectra were processed using Proteome Discoverer 1.3 software. A total of 23619 peptides (10914 specific peptides) and 2040 proteins were identified by iTRAQ analysis. Differentially expressed proteins were filtered out according to their ratio. After filtering, a total of 588 proteins, of which 300 were down- 
Fig. 2. Results of pathomorphology observations of livertoxicity in ratsafter oral administrated differentparts of Polygonum multiflorum. (A: Total group; B: Water group; C: 30 Ethanol group; D: 70 Ethanol group; E: DCM group; F: Control group).

regulated and 288 were up-regulated, were found in the total extract group; 793 proteins, of which 395 were down-regulated

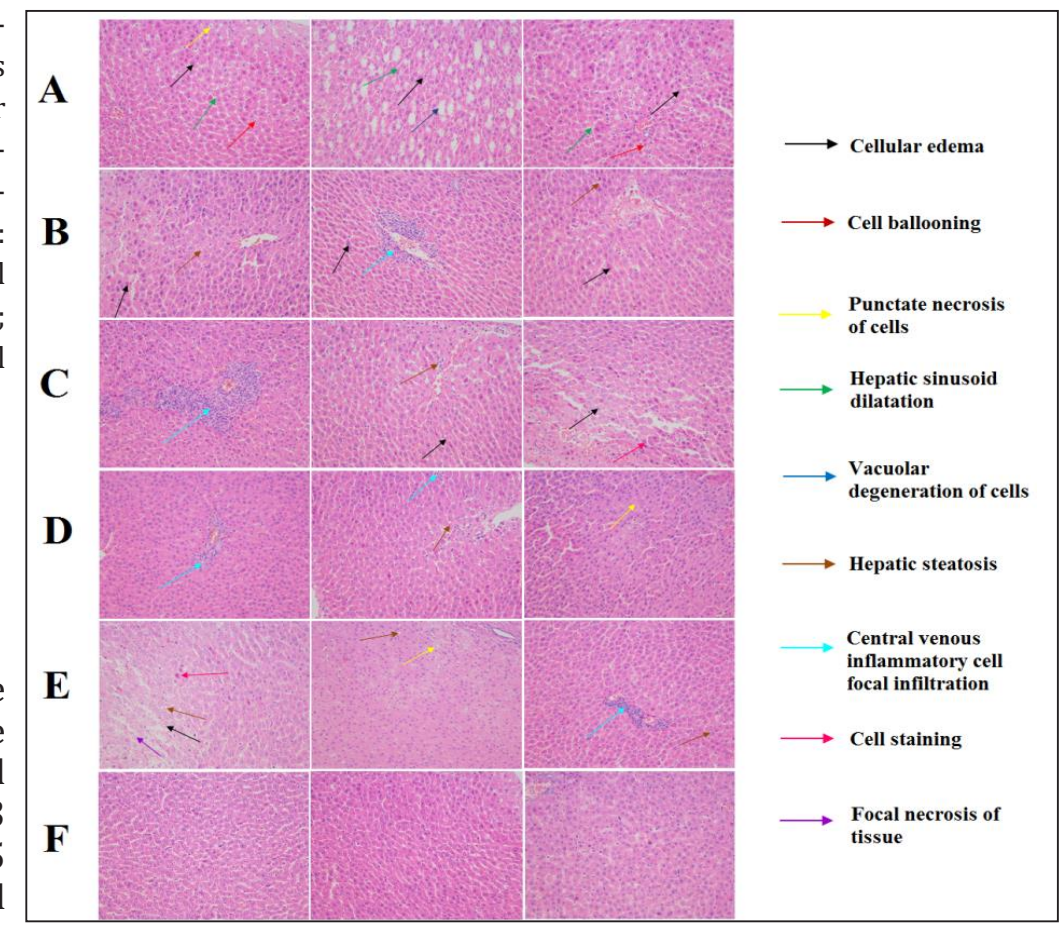
and 398 were up-

regulated in the water group; 203 different proteins, of which 105 were down-regulated and 98 were up-regulated in the 30 ethanol group; 198 proteins, of which 89 were downregulated and 109 were up-regulated in the 70 ethanol group; and 150 proteins, of which 56 were down-regulated and 94 were up-regulated in the DCM group. The above results were analysed in the next step.

These differential proteins were further analysed by the DAVID analysis tool and classified into "molecular function (MF)", "cellular component (CC)", and "biological process (BP)" subcategories. The top 20 GO categories of each group (all subcategories will be included if the total number is not up to 20) were included to determine the shared characteristics by Venn analysis, the analysis showed that (Fig. 3). The MF, CC and BP subcategories of the differential proteins involved in these five groups exhibited both similarities and differences. The BP subcategory is related with the functioning of integrated living units: cells, tissues, organs, and organisms. Three common BP were involved in these five groups, which was response to organic substance (GO:0010033), generation of precursor metabolites and energy (G0:0006091) and electron transport chain (G0:0022900). For the analysis of CC, we found that most of the differentially expressed proteins were associated with mitochondrial. There are eight common CC involved in these five groups, which was mitochondrial membrane (G0:0031966), mitochondrion (G0:0005739), organelle inner membrane(GO:0019866), cytosol(G0:0005829), envelope (GO:0031975), mitochondrial inner membrane(G0:0005743), organelle envelope(G0:0031967), mitochondrial envelope(G0:0005740). MF represented the elemental activities of a gene product at the molecular level, such as binding or catalysis. For the analysis of MF, we found that most of the differentially expressed proteins were enriched in RNA binding (GO:0003723), nucleotide binding (GO:0000166), monovalent inorganic cation transmembrane transporter activity (GO:0015077), structural molecule activity (GO:0005198) and others, as shown in Fig. 3.

Pathway enrichment analysis. To illustrate changes in biological processes, the protein expression data was mapped onto the KEGG Pathway analysis tool $[8,9]$. The top 10 Pathway of each group (all categories will be included if the total number is not up to 10) were included to determine the shared characteristics by Venn analysis, the analysis showed that (Fig. 4). The analysis showed that oxidative phosphorylation, Parkinson's disease, Huntington's disease and Alzheimer's disease pathway were involved in all five groups. 
Fig. 3. Proteins categorized as biological process (1), cellular component (2) and molecular function(3) of the five groups(Total group (A), Water group(B), 30 Ethanol group(C), 70 Ethanol group(D) and DCM group(E)). The red numbers represent the common subcategories of the groups in the Venn diagram.

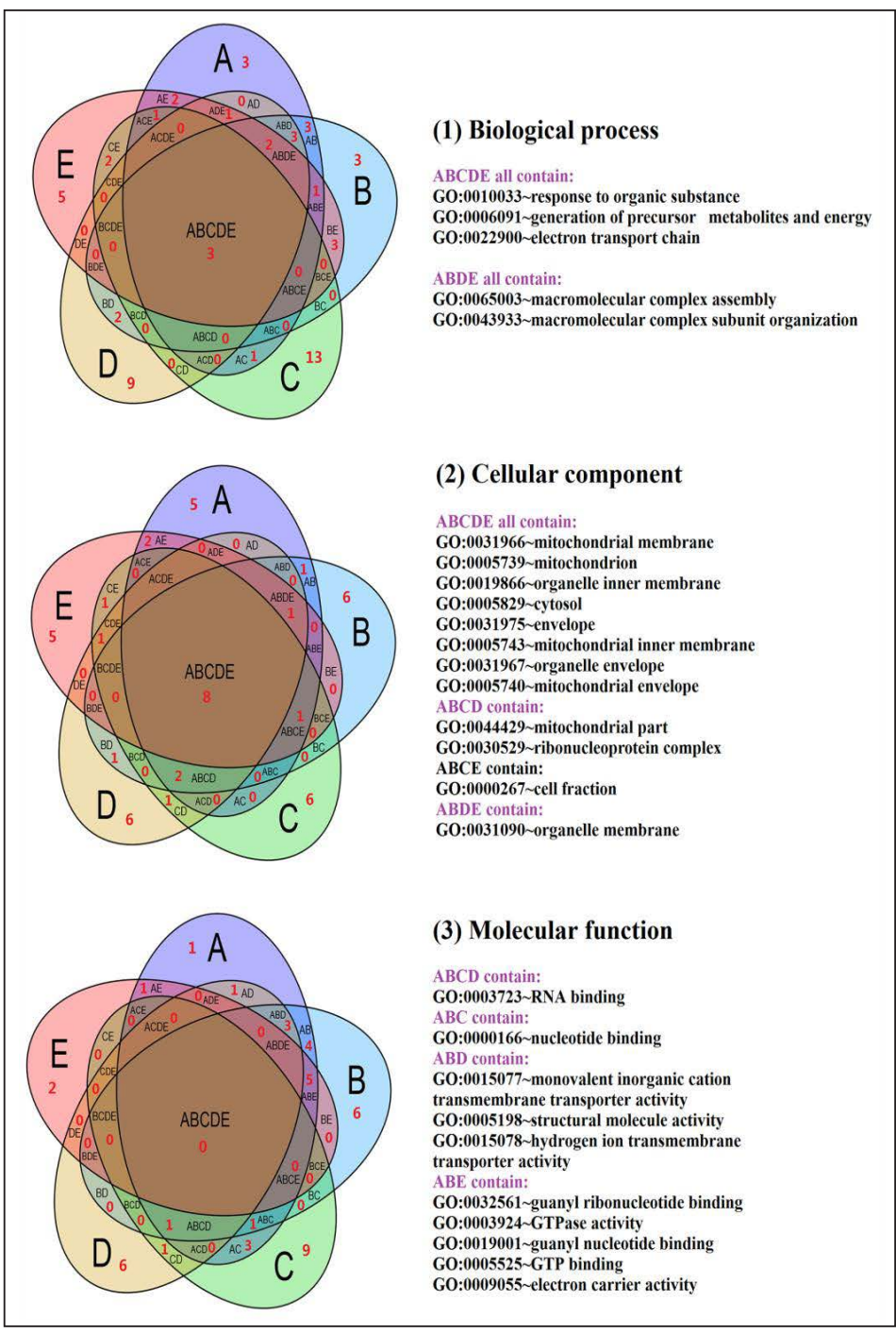

Fig. 4. The Venn diagram of pathway analysis of the five groups (Total group (A), Water group(B), 30 Ethanol group(C), 70 Ethanol group(D) and DCM group(E)). The red numbers represent the common subcategories of the groups in the Venn diagram.

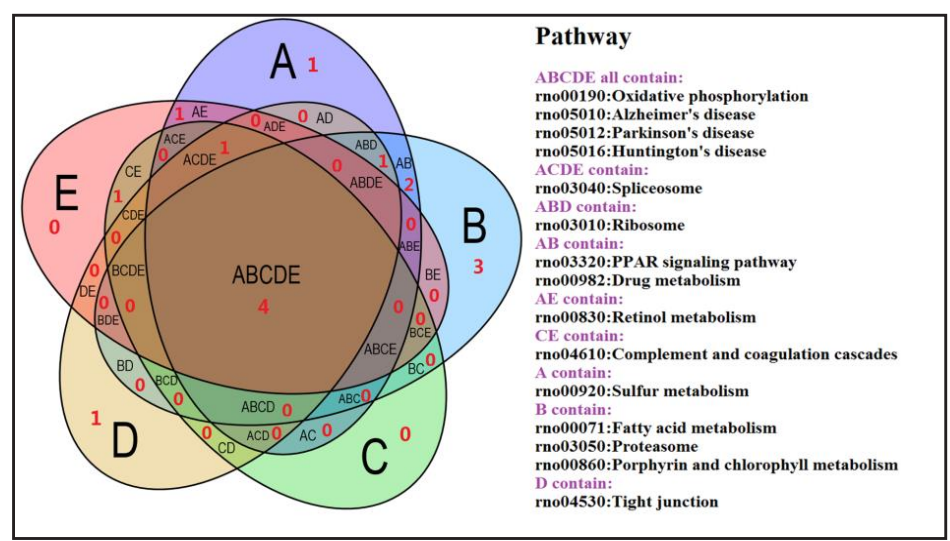

The oxidative phosphorylation pathway is directly related to drug-induced liver injury. Oxidative phosphorylation is the metabolic pathway by which cells use enzymes to oxidize nutrients, thereby releasing energy, which is used to reform ATP $[10,11]$. Liver cell apoptosis could be induced by inhibiting the complex in the mitochondrial respiratory chain and reducing the mitochondrial membrane potential (MMP)[12]. Taking the total group as an example (Fig. 4), the function of complex I is to catalyse $2 \mathrm{H}^{+}\left(\mathrm{in} \mathrm{NADH}+\mathrm{H}^{+}\right.$), which are generated by 
the tricarboxylic acid cycle (TCA), transported to the membrane space through flavin mononucleotide $(F M N)$, and then send 2 electrons to coenzyme $\mathrm{Q}(\mathrm{CoQ})$ via Fe-S. In addition, ubiquinol $\left(U_{Q} H_{2}\right)$ is generated by combining with $\mathrm{H}^{+}$, which is in the matrix, and finally with $\mathrm{CoQ}$. NADH dehydrogenase expression abnormalities can inhibit the oxidation of Fe-S clusters, ubiquinone reduction and electron transfer. Complex III, also known as cytochrome c reductase, can catalyse electron transfer from $U_{Q} H_{2}$ to $C y t c$ via Cyt $b \rightarrow F e S \rightarrow C y t c 1$ coupled with the cross membrane proton transfer. Therefore, the abnormal expression of cytochrome $\mathrm{c}$ oxidoreductase also affects electron transfer. Complex IV, also known as cytochrome c oxidase, is the final protein complex in the electron transport chain. This enzyme mediates the final reaction in the electron transport chain and transfers electrons to oxygen while pumping protons across the membrane. The final electron acceptor oxygen is reduced to water in this step. The abnormal expression of cytochrome c oxidase may affect the proton gradient. Complex V, also called ATP synthase, is the final enzyme in the oxidative phosphorylation pathway. The enzyme uses the energy stored in the proton gradient across the membrane (produced by complex I, III, IV operation process) to drive the synthesis of ATP from ADP and phosphate (Pi). Thus, the mechanism of liver injury in the total extract group may be associated with oxidative phosphorylation [13], which could be due to dysfunction of complex I, III, IV or V. This dysfunction could induce mitochondrial damage and liver cell apoptosis due to electron transfer inhibition and decreased ATP content $[14,15]$.

Fig. 4 shows that the signalling pathways of the total extract group and the water extract group were quite different compared with the other three groups. For example, the peroxisome proliferator-activated receptor (PPAR) signalling pathway, retinol metabolism, metabolism of xenobiotics via cytochrome P450 and drug metabolism were only present in these two groups. PPARs are nuclear hormone receptors that are activated by fatty acids and their derivatives [16, 17]. There are three subtypes of PPARs (PPAR- $\alpha$, $\beta / \delta$ and $\gamma$ ) that exhibit different expression patterns in vertebrates. PPAR- $\alpha$ plays a role in the clearance of circulating or cellular lipids via the regulation of gene expression involved in lipid metabolism in the liver. PPAR- $\beta / \delta$ is involved in lipid oxidation and cell proliferation and also exerts cytoprotective effects by reducing the release of IFN- $\gamma$, TNF- $\alpha$ MCP-1, IL-1 $1 \beta$, IL- 6 and other inflammatory mediators in macrophages. PPAR- $\gamma$ promotes adipocyte dif-

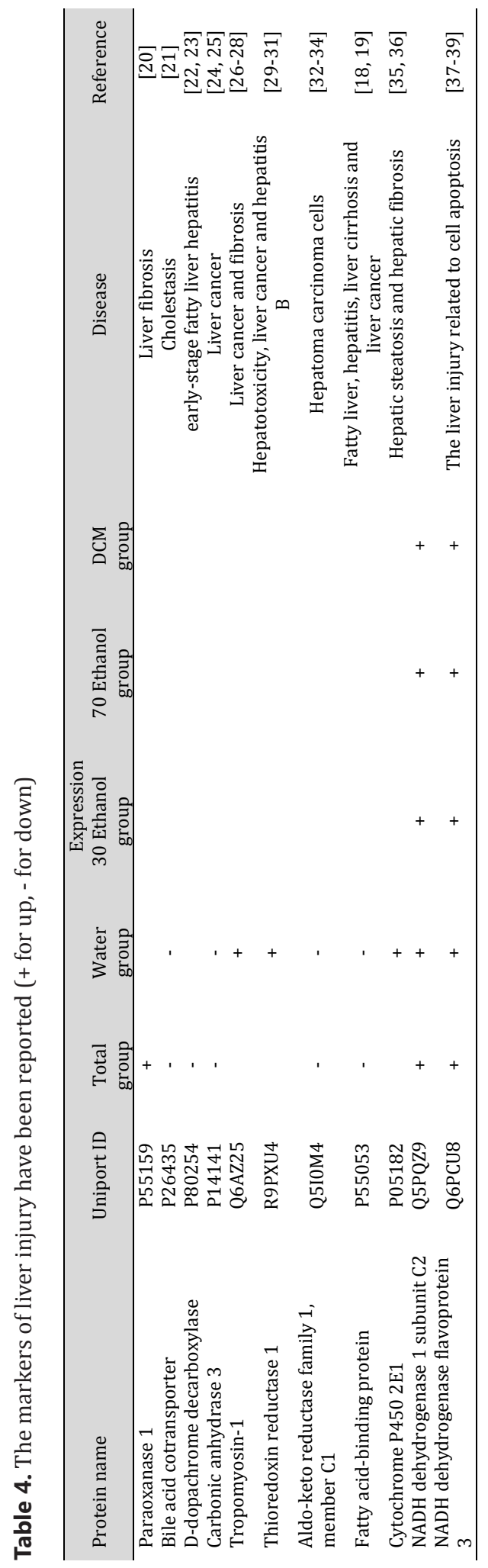




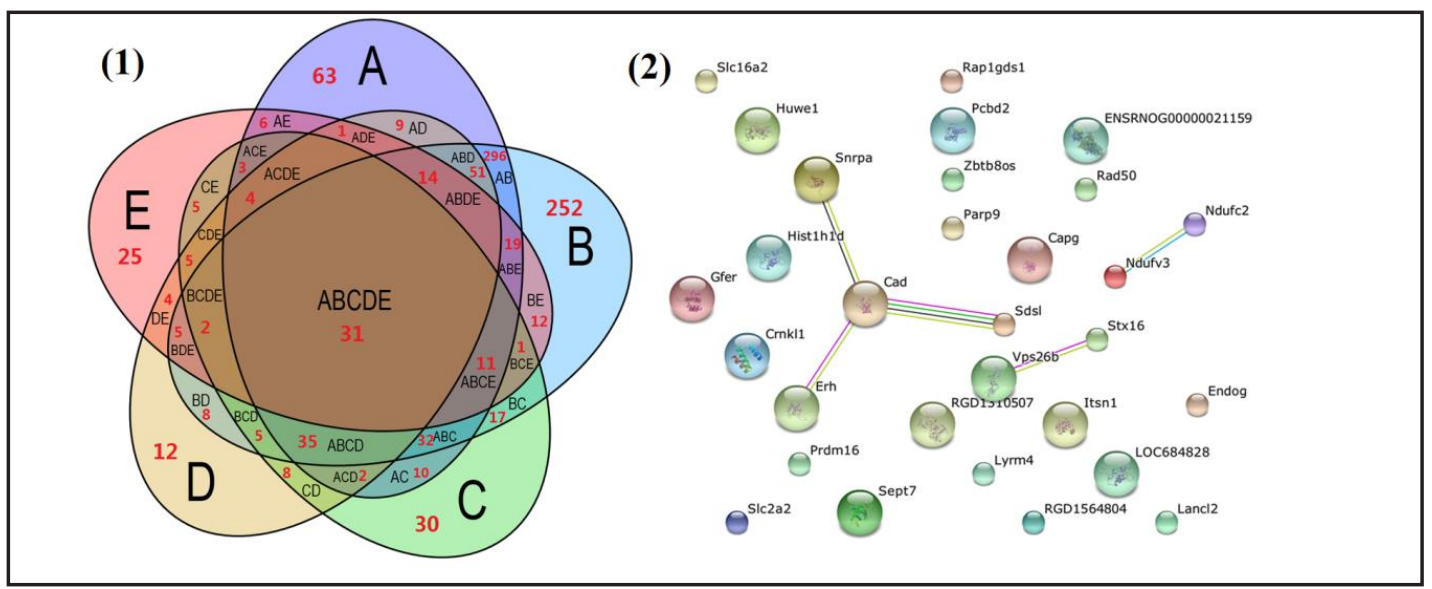

Fig. 5. (1)The cross of expressed protein in five groups, the red numbers represent the common subcategories of the groups in the Venn diagram. Total group (A), Water group(B), 30 Ethanol group(C), 70 Ethanol group(D) and DCM group(E). (2) The network of significantly differentially expressed proteins was analyzed by String software. Modes of action are shown in different colors.(Green Line-approaching; Red - gene fusion; Blue line - Co-occurrence; black line - co-expression; Purple line - experimental; Yellow line - text mining; Light blue line - database.

Fig. 6. The expression of Ndufv3 and Slc16a2 in these six group, which verified by western blot. ${ }^{*} \mathrm{P}<0.05$, comparison between the control group and treatment groups; ${ }^{* *} \mathrm{P}<0.01$, comparison between the control group and treatment groups.

ferentiation to enhance blood glucose uptake. The increased activity of PPAR- $\gamma$ in hepatic stellate cells (HSCs) could inhibit liver fibrosis and reversal of HSC proliferation via activation through reduced levels of $\alpha$-smooth muscle actin $(\alpha-S M A$, which is a fibrotic cytokine), I collagen and hydroxyproline in HSCs. The low expression of fatty acidbinding protein $(F A B P)$ in these two groups might cause liver injury [18, 19].

\section{Screening for potential hepato- toxicity biomarkers}

Potential hepatotoxicity bio-

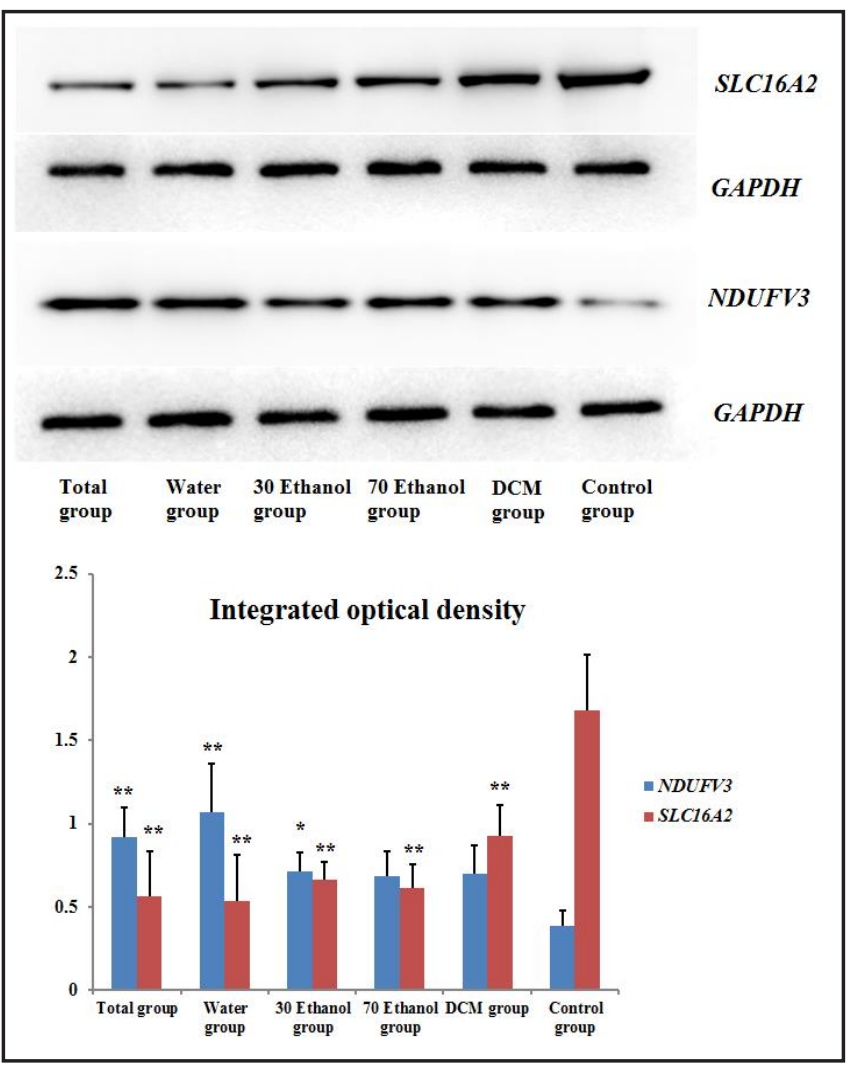

markers have been reported. Eleven proteins associated with liver damage were discovered by searching databases and existing reports. The expression of these proteins differed among the 5 administered groups owing to the differences of the 5 extracts, as shown in Table 4 . The results showed that, the biomarker associated with liver injury was existed in all administered groups, such as liver fibrosis, cholestasis, liver cancer, liver cirrhosis and others. NADH dehydrogenase 1 subunit $\mathrm{C} 2(\mathrm{Ndufc} 2)$ and NADH dehydrogenase flavoprotein 
3 (Ndufv3) were co-expressed in five administered groups. Ndufc2 and Ndufv3 belongs to $N A D H$ dehydrogenase family and mainly participates in the oxidative phosphorylation pathway. It can combine with NADH to transfer two high potential energy electrons from NADH to its FMN prosthetic group, thus oxidizing NADH and reducing FMN. Abnormal expression of these two enzymes affects the mitochondrial respiratory chain, reduces ATP content and finally leads to mitochondrion damage and cell apoptosis. This indicated the liver injury occurred in these groups. These results were also consisted with the pathway analysis.

Screening for co-expressed potential hepatotoxicity biomarkers. To determine the protein biomarkers of hepatotoxicity associated with the five groups, co-expressed proteins were screened in the liver tissues of the five groups, and the screening results are shown in Fig. 5(1). A total of 31 co-expressed proteins were screened, and the differential proteins were input into String 10.0 to analyse the protein-protein interaction network and to determine interactions among forecast functions of differential proteins. The correlation network for the forecast function of differential proteins is shown in Fig. 5(2). The pathway analysis results showed that these differential proteins participated in the digestion and absorption of spliceosomes, metabolism, oxidative phosphorylation and carbohydrate pathways, cell apoptosis and thyroid hormone signalling pathways. Ndufc2 and Ndufv3 of the NADH family were co-expressed in the livers of the five groups of rats and participated in the oxidative phosphorylation pathway. They were associated with mitochondrion damage and cell apoptosis and may be possible protein biomarkers for hepatotoxicity resulting from the different extracts of PM.

Monocarboxylic acid transport protein-8 (MCT-8/ Slc16a2) can transport specific thyroid hormone (T4) and triiodothyronine (T3), it mainly participates in the thyroid hormone signal pathway. The processes of intracellular T3 and T4 transfer were blocked due to low expression of MCT-8, and thus, the PI3K/Akt signalling pathway and Akt cannot be successfully activated, and the expression of p53 protein and caspase- 9 is not restrained, finally leading to cell apoptosis. Therefore, MCT-8 is also a possible biomarker of hepatotoxicity resulting from different extracts of PM $[40,41]$.

Validation of the results by western blot. Proteins were extracted from the livers of rats of the five treated groups and the control group. After SDS-PAGE and transfer of proteins to membranes, western blot was performed on for Ndufv3 and Slc16a2 protein. The gels of Ndufv3 and Slc16a2 are shown in Fig. 6. The results indicated that the Ndufv3 bands of the five treated groups were deeper than that of the control group, validating that the expression of Ndufv3 was up-regulated in rat livers after oral administration of PM extracts based on the grey values. In addition, expression of Slc16a2 was down-regulation in the rat livers after oral administration of PM extracts compared with the control group. The western blot results were in agreement with the iTRAQ proteomics, indicating that the two proteins are possible biomarkers of hepatotoxicity resulting from different extracts of PM.

\section{Discussion}

PM is a popular traditional Chinese medicine used in many patented drugs and prescriptions. However, the hepatotoxic components and mechanisms of liver injury resulting from PM have not been clearly elucidated even in light of widespread concerns in recent years based on reports of hepatotoxicity due to PM. In this study, the ingredients in PM were analysed by separating PM into various extracted components, and the hepatotoxicity of each extract was investigated in vivo. The results of biochemical and antioxidant indexes and histopathological analysis showed that liver injury was occurred in all 5 groups of rats administered the various extracts of PM, and all the extracts (total, water, 30 ethanol, 70 ethanol and DCM extracts) induced hepatotoxicity. The 30 ethanol group and DCM group exhibited the higher degree of hepatotoxicity than others treated groups. Emodin was the major anthraquinones component of PM was also the main component in the DCM extract, and it has been demonstrated to exert concentration- and time dependent toxic effects 


\section{Cellular Physiology Cell Physiol Biochem 2017;43:2102-2116

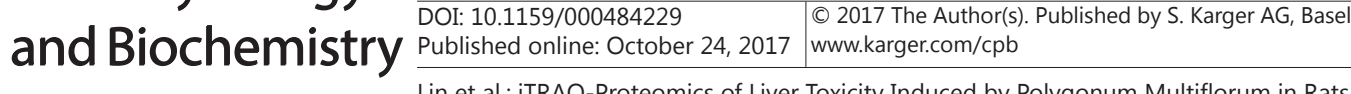

in vitro and in vivo $[42,43]$. Our previous studies proved that the 30 ethanol fraction has strongly cytotoxicity and hepatotoxicity, which was similar to the total extract based on the cell viability assay and high content screening assays. And it inferred other components in PM might be hepatotoxic [44]. These conclusions were consistent with the results of this study, and also indicated that the mechanism of liver injury induced by the total extract group may differ from that of the other groups, which might be due to the components interactions.

The levels of antioxidantindexes, such as SOD, revealed that theliver injury mechanism was possibly related to oxidative damage. The iTRAQ proteomics study showed that hepatotoxicity resulting from PM was mainly related to the abnormal activity of mitochondrion functionrelated oxidative phosphorylation pathways. Abnormal expression of NADH dehydrogenase family proteins can hinder oxidative phosphorylation, damage mitochondrion function and increase electron leakage in the respiratory chain. They could further cause the increase in ROS and the release of Cyt c, which activates caspase-9, finally resulting in cell apoptosis. The thyroid hormone signalling pathway is also related to the expression of caspase- 3 and caspase-9. The low expression of transport protein Slc16a2 caused the failure of the PI3K/Akt signalling pathway, the successful activation of Akt, the failure of $\mathrm{p} 53$ protein expression and the inhibition of caspase-9, thus resulting in cell apoptosis. The hepatotoxicity mechanism is also possibly related to the TCA cycle dysfunction owing to abnormal lipid and glucose metabolism. Furthermore, such as liver injury via bile duct blockage, jaundice hepatitis, and haemolytic diseases was occurred in total extract group. Such as the low expression and dysfunction of bile acid cotransporter lead to cholestasis [45]. This difference was possibly related to the different types and contents of ingredients in the extracts.

\section{Abbreviations}

BP (Biological process); CFDA (China Food and Drug Administration); CC (Cellular component); CoQ (Coenzyme Q); DCM (Dichloromethane); EDG (Emodin-8-0- $\beta-D-$ glucopyranoside); FABP (Fatty acid-binding protein); FDR (False discovery rate); FMN (Flavin mononucleotide); GO (Gene ontology); HE (Hematoxylin-eosin); HSCs (Hepatic stellate cells); MCT-8/ Slc16a2 (Monocarboxylic acid transport protein-8); MF (Molecular function); MHRA (Medicines and Healthcare Products Regulatory Agency); MMP (Mitochondrial membrane potential); Ndufc2 (NADH dehydrogenase 1 subunit C2); Ndufv3 (NADH Dehydrogenase flavoprotein 3); PM (Polygonum multiflorum); PPAR (Peroxisome proliferator-activated receptor); T3 (Triiodothyronine); T4 (Thyroid hormone); TCA (Tricarboxylic acid cycle); TCM (Traditional Chinese Medicine); TSG (2, 3,5, 4'-tetrahydroxystilbene-2-0- $\beta$-glucoside); UQH2 (Ubiquinol).

\section{Acknowledgements}

This work was financially supported by the National Natural Science Foundation of China (No. 81673609).

\section{Disclosure Statement}

The authors declare no competing financial interests.

\section{References}

1 Lin L, Ni B, Lin H, Zhang M, Li X, Yin X, Qu C, Ni J: Traditional usages, botany, phytochemistry, pharmacology and toxicology of Polygonum multiflorum Thunb.: a review. J Ethnopharmacol 2015;159:158-183. 


\section{Cellular Physiology Cell Physiol Biochem 2017;43:2102-2116

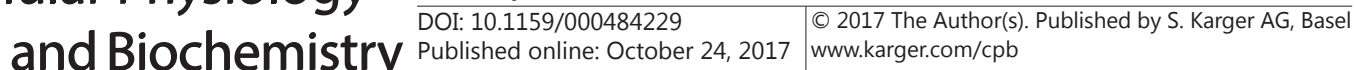

2 Dong H, Slain D, Cheng J, Ma W, Liang W: Eighteen cases of liver injury following ingestion of Polygonum multiflorum. Complement Ther Med 2014;22:70-74.

- Furukawa M, Kasajima S, Nakamura Y, Shouzushima M, Nagatani N, Takinishi A, Taguchi A, Fujita M, Niimi A, Misaka R, Nagahara H: Toxic hepatitis induced by show-wu-pian, a Chinese herbal preparation. Intern Med 2010;49:1537-1540.

4 Park GJ, Mann SP, Ngu MC: Acute hepatitis induced by Shou-Wu-Pian, a herbal product derived from Polygonum multiflorum. J Gastroenterol Hepatol 2001;16:115-117.

5 Zhang X, Hou HT, Wang J, Liu XC, Yang Q He GW: Plasma Proteomic Study in Pulmonary Arterial Hypertension Associated with Congenital Heart Diseases. Sci Rep 2016;6:36541.

-6 Pierce A, Unwin RD, Evans CA, Griffiths S, Carney L, Zhang L, Jaworska E, Lee CF, Blinco D, Okoniewski MJ, Miller CJ, Bitton DA, Spooncer E, Whetton AD: Eight-channel iTRAQ enables comparison of the activity of six leukemogenic tyrosine kinases. Mol Cell Proteomics 2008;7:853-863.

7 Lin L, Lin H, Zhang M, Ni B, Yin X, Qu C, Ni J: A novel method to analyze hepatotoxic components in Polygonum multiflorum using ultra-performance liquid chromatography-quadrupole time-of-flight mass spectrometry. J Hazard Mater 2015;299:249-259.

8 Kanehisa M, Furumichi M, Tanabe M, Sato Y, Morishima K: KEGG: new perspectives on genomes, pathways, diseases and drugs. Nucleic Acids Res 2017;45:D353-D361.

-9 Kanehisa M, Sato Y, Kawashima M, Furumichi M, Tanabe M: KEGG as a reference resource for gene and protein annotation. Nucleic Acids Research 2016;44:D457-D462.

10 Dimroth P, Kaim G, Matthey U: Crucial role of the membrane potential for ATP synthesis by F1Fo ATP synthases. J Exp Biol 2000;203:51-59.

11 Mitchell P, Moyle J: Chemiosmotic hypothesis of oxidative phosphorylation. Nature 1967;213:137-139.

12 Ling GN: Oxidative phosphorylation and mitochondrial physiology: a critical review of chemiosmotic theory, and reinterpretation by the association-induction hypothesis. Physiol Chem Phys 1981;13:29-96.

13 Syed M, Skonberg C, Hansen SH: Mitochondrial toxicity of diclofenac and its metabolites via inhibition of oxidative phosphorylation (ATP synthesis) in rat liver mitochondria: Possible role in drug induced liver injury (DILI). Toxicol In vitro 2016;31:93-102.

14 Peng X, Gan J, Wang Q Shi Z, Xia X: 3-Monochloro-1, 2-propanediol (3-MCPD) induces apoptosis via mitochondrial oxidative phosphorylation system impairment and the caspase cascade pathway. Toxicology 2016;372:1-11.

15 Syed M, Skonberg C, Hansen SH: Mitochondrial toxicity of selective COX-2 inhibitors via inhibition of oxidative phosphorylation (ATP synthesis) in rat liver mitochondria. Toxicol In vitro 2016;32:26-40.

16 Belfiore A, Genua M, Malaguarnera R: PPAR-gamma Agonists and Their Effects on IGF-I Receptor Signaling: Implications for Cancer. PPAR Res 2009;2009:835051.

17 Berger J, Moller DE: The mechanisms of action of PPARs. Ann Rev Med 2002;53:409-435.

18 Pelsers MMAL, Morovat A, Alexander GJM, Hermens WT, Trull AK, Glatz JFC: Liver fatty acid-binding protein as a sensitive serum marker of acute hepatocellular damage in liver transplant recipients. Clinical Chemistry 2002;48:2055-2057.

19 Schroeder F, Petrescu AD, Huang H, Atshaves BP, McIntosh AL, Martin GG, Hostetler HA, Vespa A, Landrock D, Landrock KK, Payne HR, Kier AB: Role of fatty acid binding proteins and long chain fatty acids in modulating nuclear receptors and gene transcription. Lipids 2008;43:1-17.

20 Marsillach J, Camps J, Ferre N, Beltran R, Rull A, Mackness B, Mackness M, Joven J: Paraoxonase- 1 is related to inflammation, fibrosis and PPAR delta in experimental liver disease. BMC Gastroenterology 2009;9:3.

21 Boyer JL, Ng OC, Ananthanarayanan M, Hofmann AF, Schteingart CD, Hagenbuch B, Stieger B, Meier PJ: Expression and characterization of a functional rat liver $\mathrm{Na}+$ bile acid cotransport system in COS-7 cells. Am J Physiol 1994;266:G382-387.

-22 Iwata T, Taniguchi H, Kuwajima M, Taniguchi T, Okuda Y, Sukeno A, Ishimoto K, Mizusawa N, Yoshimoto K: The Action of D-Dopachrome Tautomerase as an Adipokine in Adipocyte Lipid Metabolism. PloS One 2012;7:e33402.

23 Fernando H, Wiktorowicz JE, Soman KV, Kaphalia BS, Khan MF, Ansari GAS: Liver proteomics in progressive alcoholic steatosis. Toxicol Appl Pharmacol 2013;266:470-480.

24 Seymour JM, Ward K, Sidhu PS, Puthucheary Z, Steier J, Jolley CJ, Rafferty G, Polkey MI, Moxham J: Ultrasound measurement of rectus femoris cross-sectional area and the relationship with quadriceps strength in COPD. Thorax 2009;64:418-423. 


\section{Cellular Physiology Cell Physiol Biochem 2017;43:2102-2116 \begin{tabular}{ll|l} 
DOI: 10.1159/000484229 & $\begin{array}{l}\text { O } 2017 \text { The Author(s). Published by S. Karger AG, Basel } \\
\text { www.karger.com/cpb }\end{array}$
\end{tabular}}

25 Kuo WH, Chiang WL, Yang SF, Yeh KT, Yeh CM, Hsieh YS, Chu SC: The differential expression of cytosolic carbonic anhydrase in human hepatocellular carcinoma. Life Sciences 2003;73:2211-2223.

26 Perry SV: Vertebrate tropomyosin: distribution, properties and function. J Muscle Res Cell Motil 2001;22:549.

-27 Hughes JAI, Cooke-Yarborough CM, Chadwick NC, Schevzov G, Arbuckle SM, Gunning P, Weinberger RP: High-molecular-weight tropomyosins localize to the contractile rings of dividing CNS cells but are absent from malignant pediatric and adult CNS tumors. Glia 2003;42:25-35.

-28 Houle FO, Rousseau S, Morrice N, Luc M, Mongrain S, Turner CE, Tanaka S, Moreau P, Huot J: Extracellular signal-regulated kinase mediates phosphorylation of tropomyosin-1 to promote cytoskeleton remodeling in response to oxidative stress: Impact on membrane blebbing. Mol Biol Cell 2003;14:1418-1432.

29 Lincoln DT, Ali Emadi EM, Tonissen KF, Clarke FM: The thioredoxin-thioredoxin reductase system: overexpression in human cancer. Anticancer Res 2003;23:2425-2433.

30 Han P, Ji D, Chen GF, Li YG, Liu Y: Upregulation of thioredoxin reductase 1 gene expression by small surface protein of hepatitis B. Chin J Gastroenterol Hepatol 18:344-347.

31 Hu W, Fang ZQ, Liang C, Guan DY, Wu ZH: RNA Interference Studying the Role of TrxR1 Gene in Proliferation of Hepatocarcinoma Cells and Adjustment of Several TCM Therapeutical Principles. Lishizhen Med Materia Medica Res 2012;23:192-194.

32 Cao D, Fan ST, Chung SS: Identification and characterization of a novel human aldose reductase-like gene. J Biol Chem 1998;273:11429-11435.

33 de la Fuente JA, Manzanaro S, Martin MJ, de Quesada TG, Reymundo I, Luengo SM, Gago F: Synthesis, activity, and molecular modeling studies of novel human aldose reductase inhibitors based on a marine natural product. J Med Chem 2003;46:5208-5221.

34 Yang XD, Tang DN, Wang J, Cao DL: [Screening of the drug resistance-associated gene in HepG2 cell line transfected with aldose reductase like gene-1 (ARL-1)]. Ai Zheng 2003;22:1289-1295.

-35 Lu YK, Wang XD, Cederbaum AI: Lipopolysaccharide-induced liver injury in rats treated with the CYP2E1 inducer pyrazole. Am J Physiol Gastrointestl Liver Physiol 2005;289:G308-G319.

-36 Nieto N, Cederbaum AI: Increased Sp1-dependent transactivation of the LAMgamma 1 promoter in hepatic stellate cells co-cultured with HepG2 cells overexpressing cytochrome P450 2E1 J Biol Chem 2003;278:15360-15372.

-37 Kopylchuk GP, Voloshchuk OM: [NADH:ubiquinone reductase and succinate dehydrogenase activity in the liver of rats with acetaminophen-induced toxic hepatitis on the background of alimentary protein deficiency]. Ukr Biochem J 2015;87:121-126.

38 Kucera O, Endlicher R, Rousar T, Lotkova H, Garnol T, Drahota Z, Cervinkova Z: The effect of tert-butyl hydroperoxide-induced oxidative stress on lean and steatotic rat hepatocytes in vitro. Oxid Med Cell Longev 2014;2014:752506.

39 Voloshchuk ON, Kopylchuk GP: [Activity of liver mitochondrial NAD+-dependent dehydrogenases of the krebs cycle in rats with acetaminophen-induced hepatitis developed under conditions of alimentary protein deficiency]. Biomed Khim 2016;62:169-172.

40 Gibson EM, Henson ES, Haney N, Villanueva J, Gibson SB: Epidermal growth factor protects epithelialderived cells from tumor necrosis factor-related apoptosis-inducing ligand-induced apoptosis by inhibiting cytochrome c release. Cancer Res 2002;62:488-496.

41 Zhou HL, Li XM, Meinkoth J, Pittman RN: Akt regulates cell survival and apoptosis at a postmitochondrial level. J Cell Biol 2000;151:483-494.

42 Li CL, Ma J, Zheng L, Li HJ, Li P: Determination of emodin in L-02 cells and cell culture media with liquid chromatography-mass spectrometry: application to a cellular toxicokinetic study. J Pharm Biomed Anal 2012;71:71-78.

43 National Toxicology P: NTP Toxicology and Carcinogenesis Studies of EMODIN (CAS NO. 518-82-1) Feed Studies in F344/N Rats and B6C3F1 Mice. Natl Toxicol Program Tech Rep Ser 2001;493:1-278.

44 Lin L, Li H, Lin H, Zhang M, Qu C, Yan L, Yin X, Ni J: A New Perspective on Liver Injury by Traditional Chinese Herbs Such As Polygonum multiflorum: The Geographical Area of Harvest As an Important Contributory Factor. Front Pharmacol 2017;8:349.

45 Ho RH, Leake BF, Robertson RL, Lee W, Kim RB: Ethnicity-dependent polymorphism in Na+-taurocholate cotransporting polypeptide (SLC10A1) reveals a domain critical for bile acid substrate recognition. J Biol Chem 2004;279:7213-7222. 